\title{
Standard framework and experience of living donor liver transplantation for overseas non-Korean patients at Asan Medical Center
}

Sang-Hoon Kim, Shin Hwang, Gi-Won Song, Dong-Hwan Jung, Chul-Soo Ahn, Ki-Hun Kim, Deok-Bog Moon, Tae-Yong Ha, Gil-Chun Park, Sung-Gyu Lee

Department of Surgery, Asan Medical Center, University of Ulsan College of Medicine, Seoul, Korea

Background: Liver transplantation (LT) for foreign patients is a sensitive issue because of the possibility of transplant tourism and the difficulty in follow-up. This study describes the standard framework and experience of living donor LT (LDLT) for overseas non-Korean patients in a Korean high-volume LDLT center.

Methods: The framework and experience of LDLT for 105 non-Korean patients from 2010 to 2019 were retrospectively investigated. Results: Only 3.1\% of patients who underwent LDLT were overseas non-Koreans; of these, 83.8\% were from the United Arab Emirates and Mongolia. Selection criteria for recipients and donors were the same as for Korean citizens. Of the 105 recipients, 95 (90.5\%) were adults. The most common reasons for transplantation were hepatitis B or C virus-associated liver cirrhosis. Of the 95 adults, $78(82.1 \%)$ received right liver grafts, and $16(16.8 \%)$ received dual grafts. The most frequent donors for adult recipients were sons and daughters, whereas the most frequent donors for pediatric recipients were parents. Of the 10 pediatric patients, eight were from the United Arab Emirates; their common primary diseases were biliary atresia, acute liver failure, hepatoblasto$\mathrm{ma}$, and genetic metabolic diseases. The 1-, 3-, and 5-year posttransplant overall patient survival rates in all patients were $96.2 \%$, $92.4 \%$, and $92.4 \%$, respectively. The 5 -year overall patient survival rates were $91.8 \%$ in adult recipients and $100 \%$ in pediatric recipients $(P=0.47)$.

Conclusions: LDLT at Korean high-volume LT centers including our institution is safe and effective for non-Korean patients with end-stage liver disease seeking alternatives not available in their own countries.

Corresponding author: Shin Hwang

E-mail: shwang@amc.seoul.kr

(c) The Korean Society for Transplantation

This is an Open Access article distributed under the terms of the Creative Commons Attribution Non-Commercial License (http://creativecommons.org/licenses/by-nc/4.0/) which permits unrestricted non-commercial use, distribution, and reproduction in any medium, provided the original work is properly cited. 